\title{
The use of hormonal replacement therapy and the risk of stroke and myocardial infarction in women
}

\author{
S G THOMPSON, T W MEADE, AND G GREENBERG* \\ From the MRC Epidemiology and Medical Care Unit, Northwick Park Hospital, Harrow, Middlesex, UK.
}

\section{ABSTRACT}

Study objective: To determine whether there is an association between the use of hormonal replacement therapy (HRT) and the risk of stroke and myocardial infarction (MI).

Design: A case-control study of women with stroke or MI was undertaken.

Setting: The cases were notified from 83 general practices to the coordinating centre at Northwick Park Hospital, where the diagnoses were independently confirmed.

Subjects: The cases, 603 white women aged 45-69, were each matched to two controls for age and general practitioner. Of the controls, $79 \%$ were the first eligible, $15 \%$ the second eligible and $6 \%$ were obtained at the third or more attempt.

Measurements and main results: A research nurse completed a questionnaire for each case and both controls, which included information obtained from the medical notes on the prescriptions of HRT. An independent quality control check on the selection of controls and on the abstraction of information from the medical notes was made.

More than one HRT prescription had been given to 109 cases $(18 \%)$ and 174 controls $(14 \%)$, showing a weak association between the risk of stroke and MI and the past use of HRT (relative risk [RR] 1.36, 95\% confidence interval [CI] 1.01-1.81). There was a stronger association with preparations contained progestogen alone (RR 1.90, 95\% CI 1.11-3.25). On average, HRT had been used 9 years before recruitment to the study and for 15 months. However, the observed gradients of risk according to the duration of HRT use and time since HRT use do not support a causal interpretation. Also, the estimated relative risks were reduced when allowance was made for other cardiovascular risk factors.

Conclusions: There is no evidence that the use of HRT as recently prescribed in the UK constitutes a major cardiovascular risk or benefit.

Current evidence about the potential risks of cardiovascular disease (CVD) resulting from the use of hormonal replacement therapy (HRT) is equivocal. Evidence suggesting that oestrogen reduces CVD risks includes the lower risk of CVD in premenopausal women compared to postmenopausal women of the same age ${ }^{1}$ and the increased CVD risk associated with a surgical menopause. ${ }^{23}$ However, oestrogen has an adverse effect on the risk of CVD in other situations, for example in women using oral contraceptives ${ }^{4}$ and in men receiving oestrogen therapy..$^{5-7}$ Direct evidence on the CVD risks associated with the use of noncontraceptive oestrogen has come from both prospective and case-control epidemiological studies. However, the results of some of the most recent studies

* Present address: Department of Health, Richmond House, 79 Whitehall, London SWIA 2NS. have been in conflict with each other ${ }^{8}$ in showing a reduction in risk ${ }^{9}$ or an increase in risk. ${ }^{10}$

Most of the epidemiological studies yet available have primarily investigated HRT containing oestrogen alone. HRT containing progestogen alone, or combined oestrogen and progestogen, has received little attention and may have different effects. For example; some studies have suggested that the progestogenic component of oral contraceptives may increase the risk of stroke and ischaemic heart disease. ${ }^{1112}$ Also, as the epidemiological studies performed so far have been mainly based in the USA, the relevance of these to women in the UK is uncertain. For example, the ages of women receiving HRT, the doses and schedules used and, in particular, the duration of HRT use may be substantially different in the UK than in the USA. The use of combined preparations in the UK has been increasing 
over the past few years following the reduction in the late 1970s in the use of unopposed oestrogen, which was shown to increase the risk of endometrial cancer. ${ }^{13}$

The case-control study described here was planned with these considerations in mind. The aim was to investigate the association between the use of HRT, both overall and separately according to progestogen and oestrogen content, and the incidence of stroke and myocardial infarction (MI) in women aged 45-69 years in the UK. It was decided to base the study in general practice, since those data would be more representative of HRT use overall than data from special menopause clinics.

\section{Methods}

The study was based in 83 of the general practices belonging to the Medical Research Council's General Practice Research Framework. ${ }^{14}$ At each practice, cases of fatal or non-fatal stroke or MI in white women aged 45 to 69 were notified to the coordinating centre at Northwick Park. In order to increase the numbers of cases available, the first part of the study, carried out in September 1982, involved the retrospective retrieval of information on such cases for the 1 year period before that date. In the second part of the study, carried out from September 1982 to January 1986 , suspected cases were notified to the coordinating centre as they arose. Fatal cases tended not to be fully represented in the first part of the study because the medical notes had often already been returned to the Family Practitioner Committees.

The coordinating centre followed up all notifications of suspected stroke or MI and obtained, where possible, copies of hospital records, clinical and laboratory test results and autopsy findings. The classification of events by a single assessor ignorant of the woman's drug history, was carried out according to WHO criteria. ${ }^{1516}$ The "definite" and "possible" categories for MI were combined for the purpose of statistical analysis, while suspected cases not meeting the WHO criteria were excluded from the study.

A research nurse in each practice completed a questionnaire for each case patient and for two controls. Information obtained from the medical notes included recorded prescriptions of HRT and the past medical history. HRT was defined on the basis of a list of 76 preparations drawn up before the study began, six of which (Harmogen, Mixogen, Premarin, Primolut N, stilboestrol and ethinyl oestradiol) accounted for $60 \%$ of the recorded prescriptions. Information obtained directly from the subjects (or their next of kin) by interview included reproductive history, oral contraceptive use, smoking habit, family history of stroke and MI, and menopausal status. For a proportion of those cases with a fatal event, a number of these latter items of information were not available. A woman was classified as premenopausal if she was still menstruating, whether regularly or not. The two controls were caucasian women matched to the case for age (to within 2 years) and for general practitioner. The controls were selected by working through the age/sex register, starting from the name of the case patient, taking the first eligible older and the first eligible younger patient. It was also a requirement, for both cases and controls, that the medical notes for their adult life were complete.

After about 250 cases had been recruited, a formal assessment of the power of the study (taking into account only the rate of ever use of HRT observed in controls at that time) led to the decision to recruit a total of about 600 cases in all. It was estimated that a study of 600 cases (and 1200 controls) would have a power of about $85 \%$ to detect, as significant at the $5 \%$ level, true relative risks associated with ever use of any HRT further from unity than 1.4 or 0.7 . Such a study would also have a power of about $85 \%$ to detect true relative risks associated with the ever use of HRT containing progestogen alone, further from unity than 1.8 or 0.45 .

Specially trained quality control (QC) nurses travelled to each of 83 general practices and checked the progress of the study with the research nurse at the practice. The procedure for the selection of controls was checked for two to four randomly chosen controls at each practice. Of 215 controls checked overall, only four (all in one centre) had been selected in a way likely to invalidate comparability of cases and controls. The controls in this centre were abandoned and new ones sought. The QC nurse also made a clieck on the abstraction of data from the medical notes, in particular by filling in the section of the form on HRT use. For the women who had been noted to use HRT by either the practice nurse or the QC nurse, $80 \%$ of the forms were in agreement, $15 \%$ were in disagreement when one of the nurses had noted a single HRT prescription (and the other had noted none), but only $4 \%$ were in disagreement when one of the nurses had noted two or more HRT prescriptions. A single HRT prescription would presumably reflect a generally short duration of HRT use by the woman concerned (if she even in fact used the prescription at all). For this reason, and because the quality control exercise revealed that such prescribing was not necessarily detected reliably, it was decided to compare cases and controls with respect to HRT use, which was defined as having received at least two HRT prescriptions.

The relative risks of stroke and MI according to HRT use were estimated using conditional maximum likelihood logistic regression, ${ }^{17}$ appropriate for 
matched case-controls studies. The $p$ values quoted are two sided, and are given exactly for values between 0.2 and 0.001 .

\section{Results}

A total of 603 confirmed cases (244 strokes and 359 MIs), together with 1206 controls, were recruited to the study. Of the 148 cases recruited in the first retrospective part of the study, $5(3 \%)$ had suffered fatal events, while of the 455 cases recruited in the second part of the study, $126(28 \%)$ suffered fatal events. Of the 359 cases of MI, $84(23 \%)$ were assigned the WHO category of "possible" rather than "definite".

Of the controls finally selected, $79 \%$ were the first eligible control, $15 \%$ the second eligible control and $6 \%$ were obtained only after the failure of two or more previous attempts to find controls. The main reasons for failure to select eligible controls were that the women did not attend for interview ( $58 \%$ of failures), that the women were unable to attend $(9 \%)$ or that the women had moved from the practice $(8 \%)$.

Fourteen per cent of controls and $18 \%$ of cases had received more than one HRT prescription; $7 \%$ of each were recorded as having received a single HRT prescription (table 1). The relative risk of 1.36 (table 1) associated with receiving more than one HRT prescription was statistically significant $(p=0.04)$; it did not differ significantly $(p>0.2)$ either between stroke and MI cases (relative risks of 1.20 and 1.48, respectively) or between fatal and non-fatal events. Only 17 (4 cases and 13 controls), or $1 \%$, of the women overall were using HRT at the time of the case's stroke or MI, so the possibility of detecting a risk or benefit associated with current use of HRT (rather than with past use) was small.

For those women who had received more than one HRT prescription, the average estimated duration of HRT use was 15 months. The distribution of the duration of use was skew, the median being 7 months. The average time since HRT was last used was 9 years.
There were trends in the risk of stroke and MI (table 2) according to duration of use $(p=0.09)$ and time since HRT use $(p=0.009)$, such that the greatest estimated risks were observed in those women who had used HRT only for a shorter period of time or had used HRT a longer time ago. These observations do not support the hypothesis that HRT use is a cause of stroke or MI. No significant trend of the risks associated with HRT use according to the age of the women was observed $(p>0 \cdot 2)$.

Of the cases and controls overall, $11 \%$ had been prescribed HRT containing oestrogen alone on more than one occasion, the percentages for HRT containing progestogen alone and combined oestrogen-progestogen preparations being $4 \%$ and $2 \%$ respectively. The use of oestrogen (either alone, or in combination with progestogen) was not significantly associated with the risk of stroke and MI (both $p>0.2$, table 3). However the use of HRT containing progestogen alone was significantly associated with the risk of stroke and MI, with an estimated relative risk of $1.90(p=0.02$, table 3$)$. The corresponding estimated relative risk was 5.27 for fatal events and 1.46 for non-fatal events $(p=0.08$ for a difference between fatal and non-fatal events), but there was no indication of any difference between strokes and MIs $(p>0.2)$. An investigation of the trend of the risk according to duration of use of HRT containing progestogen alone suggested a decreasing risk with increasing duration $(\mathrm{p}=0.08)$, which does not support a causal hypothesis.

A previous study of new HRT users ${ }^{18}$ has suggested that smoking may be an important confounding factor that needs to be taken into account in studies of HRT and cardiovascular disease. Indeed in this study, smoking was related to the risk of stroke and MIcurrent cigarette smokers had a relative risk of 2.47 $(p<0.001)$ compared to women who had never smoked cigarettes-and the proportion of women who had used HRT was greater in current and past cigarette smokers $(17 \%)$ than in those who had never smoked (13\%). Menopausal status was not

Table 1 Numbers of cases and controls, and estimates of relative risk of stroke and myocardial infarction according to the number of recorded prescriptions of hormone replacement therapy (HRT)

\begin{tabular}{|c|c|c|c|c|c|c|}
\hline & \multicolumn{4}{|c|}{ Numbers (\%) of: } & \multirow[b]{2}{*}{ Relative risk* } & \multirow[b]{2}{*}{ (95\% confidence interval) } \\
\hline & Cases & & Contr & & & \\
\hline \multicolumn{7}{|l|}{ HRT prescriptions: } \\
\hline No prescription & 453 & (75) & 945 & (78) & 1 & \\
\hline One prescription & 41 & (7) & 87 & (7) & 1.02 & $(0 \cdot 68,1 \cdot 53)$ \\
\hline More than one prescription & 109 & (18) & 174 & (14) & $1 \cdot 36$ & $(1 \cdot 01,1 \cdot 81)$ \\
\hline Total & 603 & $(100)$ & 1206 & $(100)$ & & \\
\hline
\end{tabular}

- Relative risks, relative to the group of women with no HRT prescription, take into account the matched design of the study. 
Table 2 Numbers of cases and contiols who had received more than one prescription for $H R T$, and estimates of relative risk of stroke and myocardial infarction, according to (i) duration of HRT use and (ii) length of time since hormone replacement therapy use

\begin{tabular}{lll}
\hline & Numbers (\%) of: \\
\cline { 2 - 3 } Time period & Cases Controls & Relative risk* \\
\hline
\end{tabular}

\begin{tabular}{lrllll}
\multicolumn{5}{c}{ (i) Duration of use } \\
1-3 months & 41 & $(38)$ & 41 & $(24)$ & 2.14 \\
$4-6$ months & 19 & $(17)$ & 39 & $(22)$ & 1.09 \\
$7-12$ months & 18 & $(17)$ & 37 & $(21)$ & 1.06 \\
$13-24$ months & 15 & $(14)$ & 28 & $(16)$ & 1.14 \\
$>24$ months & 16 & $(15)$ & 29 & $(17)$ & 1.19 \\
Total & 109 & $(100)$ & 174 & $(100)$ \\
& & \multicolumn{5}{c}{$p$ value for trend $=0.09$}
\end{tabular}

(ii) Time since use

$\begin{array}{cccccc}0-1 \text { years } & 13 & (12) & 26 & (15) & 1.06 \\ 2-5 \text { years } & 15 & (14) & 41 & (24) & 0.74 \\ 6-10 \text { years } & 32 & (29) & 53 & (30) & 1.34 \\ 11-15 \text { years } & 23 & (21) & 29 & (17) & 1.73 \\ >15 \text { years } & 26 & (24) & 25 & (14) & 2.44 \\ \text { Total } & 109 & (100) & 174 & (100) & \end{array}$

$p$ value for trend $=0.009$

- Relative risks, relative to the group of women with no HRT prescription, take into account the matched design of the study.

significantly associated with the risk of stroke and MI, the relative risk being $1.10(\mathrm{p}>0.2)$ comparing postmenopausal to premenopausal women, although only $6 \%$ of the women overall were classified as premenopausal. Smoking habit and other personal characteristics were unknown for a substantial proportion of the women who had suffered fatal events; these women (and their corresponding controls) had to be omitted in analyses which adjusted for potential confounding factors. This process of omission by itself reduced the estimated relative risks associated with the use of HRT, or with the use of HRT containing progestogen alone (table 4). Allowance for a number of potential confounding factors further reduced the estimated relative risk associated with the use of HRT containing progestogen alone (table 4). No type of HRT use was significantly associated with the risk of stroke and MI, independently of the potential confounding factors (all $\mathrm{p}>0 \cdot 2$, table 4).

\section{Discussion}

The extent of HRT use in the UK as revealed by this study was quite limited. Only $16 \%$ of all the women in the study were recorded as having received more than one prescription for HRT, and their average duration of use was only 15 months. Only $1 \%$ of the women overall were currently using HRT; overall, the average time since last use of HRT was 9 years. Thus perhaps it is not surprising that no strong evidence of cardiovascular risk or benefit associated with (the mainly past) use of HRT was demonstrated. Such elevated relative risks as were estimated in this study were not maintained in an analysis that adjusted for potential confounding factors. Moreover, the trends of risk observed, according to duration of HRT use and time since last use, do not favour a causal interpretation. Numbers did not allow the separate analysis of those MIs in the "possible" WHO category; nor was it possible to classify reliably strokes as haemorrhagic or thrombotic.

Potential confounding factors that need to be considered in studies of HRT are age, ethnic group, menopausal status, smoking, past medical history and social class. In the present study, controls were matched for age and all the women were white. Within the age group overall, little effect of menopausal status on the risk of stroke and MI was evident. Information on smoking habit obtained at interview and past medical history obtained from the medical notes was available and was used in the analysis providing

Table 3 Numbers of cases and controls who had received more than one precription of oestrogen alone, progestogen alone or combined oestrogen-progestogen hormone replacement therapy preparations, and corresponding estimates of relative risk of stroke and myocardial infarction

Numbers (\%) receiving more than one prescription

Cases Controls Relative risk* (95\% confidence interval)

\begin{tabular}{lcccccc}
\hline $\begin{array}{l}\text { Type of HRT: } \\
\text { Oestrogen alone }\end{array}$ & 68 & $(11)$ & 127 & $(11)$ & $1 \cdot 12$ & $(0 \cdot 79,1 \cdot 57)$ \\
$\begin{array}{l}\text { Progestogens alone } \\
\begin{array}{l}\text { Combined oestrogen- } \\
\text { progestogen }\end{array}\end{array}$ & 30 & $(5)$ & 34 & $(3)$ & 1.90 & $(1 \cdot 11,3 \cdot 25)$ \\
\hline
\end{tabular}

* Relative risks, relative to the group of women with no prescription for the corresponding type of HRT, take into account the matched design of the study. 
Table 4 Estimated relative risks* of stroke and myocardial infarction, associated with having received more than one hormone replacement therapy (HRT) prescription, with and without adjustment for potential confounding factors

\begin{tabular}{|c|c|c|c|c|}
\hline & \multirow{2}{*}{$\frac{\text { All women }}{\text { Not adjusted }}$} & \multicolumn{3}{|c|}{ Restricted to women with known confounding factorst } \\
\hline & & Not adjusted & Adjustedt & ( $95 \%$ confidence interval) \\
\hline \multicolumn{5}{|l|}{ More than one HRT prescription of: } \\
\hline Any HRT & 1.36 & 1.26 & 1.29 & $(0 \cdot 82,2 \cdot 00)$ \\
\hline Oestrogen alone HRT & $1 \cdot 12$ & $1 \cdot 11$ & 1.09 & $(0 \cdot 65,1 \cdot 82)$ \\
\hline Progestogen alone HRT & 1.90 & 1.35 & 1.02 & $(0 \cdot 45,2 \cdot 32)$ \\
\hline Combined oestrogen-progestogen HRT & 0.86 & 0.90 & $1 \cdot 16$ & $(0 \cdot 43,3 \cdot 12)$ \\
\hline
\end{tabular}

- Relative risks, relative to the group of women with no prescription for the corresponding type of HRT, take into account the matched design of the study.

t The confounding factors adjusted for are marital status, smoking habit, family history of MI, and past medical histories of: hypertension, venous thrombosis, stroke, myocardial infarction and diabetes. At least one of these was unknown for 162 cases and 10 controls.

adjusted relative risk estimates (table 4). Social class was not available in this study; it is possible that women of higher social class tend to use HRT more than other women and that their risk of cardiovascular disease is lower. If this were so, the relative risk estimates observed in this study would be biased downwards. It is also possible that adjustment for the past medical history of certain diseases actually overcompensates for these as potential confounding factors, since some of these past histories may reflect the biological pathways by which the cardiovascular events in this study came about. For the same reason, the adjustment for HDL cholesterol levels in the Framingham report ${ }^{10}$ seems inappropriate since HDL cholesterol is a likely mediator of the effect of oestrogen. ${ }^{19} 20$

In the present study, while $11 \%$ of the women overall had been prescribed HRT containing oestrogen alone (on at least two occasions), some $4 \%$ and $2 \%$ had been prescribed combined oestrogenprogestogen and progestogen alone preparations respectively. Most previous epidemiological studies on cardiovascular disease and the use of postmenopausal HRT are American studies of preparations containing oestrogen alone, and mainly concern women who had used HRT for longer or more recently than those in the present study. A number of these studies (of which there have been over 15 ) have shown protective effects of postmenopausal oestrogen use on the risk of CVD (that is, a relative risk estimate below unity), while a few others have shown near null effects (that is, a relative risk estimate close to unity). ${ }^{21}$ Only two sources of data suggest increased risk. First, two small studies $22{ }^{23}$ based on 19 and 17 cases of non-fatal MI in the same population gave unrealistically high estimated relative risks of 9.3 and 7.5 respectively. The high proportion of the cases that were smokers and the large loss of study participants that occurred make the interpretation of these studies difficult. Secondly, data from the Framingham Study at first suggested an adverse effect of postmenopausal oestrogen on cardiovascular risk, ${ }^{10}$ but a later reanalysis, ${ }^{24}$ restricting the outcomes included to non-fatal MIs and fatal coronary heart disease (excluding coronary insufficiency and angina pectoris considered previously), showed a protective effect of oestrogen use in women aged 50-59. Taking this evidence as a whole, therefore, it seems that the use of HRT containing oestrogen alone may quite possibly exert a beneficial effect on cardiovascular risk. ${ }^{21}$ However, it is not necessarily appropriate to extrapolate from these studies to the use of HRT containing progestogen as prescribed in the UK. The different doses and potencies of oestrogen that have been used in, for example, the treatment of prostatic cancer in men and in oral contraceptives may explain the different and adverse effects of oestrogen on CVD risk experienced in other situations.

It must be concluded from the present study that there is no evidence that the use of HRT as recently prescribed in the UK constitutes a major cardiovascular risk or benefit. It should be noted that the women who had used HRT in the present study had used it, on average, only for 15 months some 9 years before. More evidence is certainly required on the use of HRT containing progestogen, since the frequency and duration of use of such preparations may well increase over the next few years, particularly as a treatment for postmenopausal osteoporosis.

We thank the doctors and nurses in the MRC General Practice Research Framework who participated in this study, and we are very grateful to Professor Hugh Tunstall-Pedoe who undertook the substantial task of assessing the cases of stroke and MI. We are also grateful to Pat O'Kelly for the clerical and data processing work involved in the study.

The contents of this article represent the authors' views alone and do not commit the Department of Health.

Address for correspondence and reprints: $\mathbf{S}$ G Thompson, Department of Clinical Epidemiology and General Practice, Royal Free Hospital Medical School, Rowland Hill Street, London NW3 2PF. 


\section{References}

${ }^{1}$ Gordon T, Kannel WB, Hjortland MC, McNamara PM. Menopause and coronary heart disease: The Framingham Study. Ann Intern Med 1978; 89: 157-61.

2 Robinson RW, Higano N, Cohen WD. Increased incidence of coronary heart disease in women castrated prior to the menopause. Arch Intern Med 1959; 104: 908-13.

${ }^{3}$ Colditz GA, Willett WC, Stampfer MJ, Rosner B, Speizer FE, Hennekens $\mathrm{CH}$. Menopause and the risk of coronary heart disease in women. N Engl J Med 1987; 316: 1105-10.

4 Royal College of General Practitioners' Oral Contraceptive Study. Mortality among oral contraceptive users. Lancet 1977; ii: 727-31.

${ }^{5}$ Blackard CE, Doe RP, Mellinger GT, Byar DP. Incidence of cardiovascular disease and death in patients receiving diethylstilbestrol for carcinoma of the prostate. Cancer 1970; 26: 249-56.

${ }^{6}$ The Coronary Drug Project Research Group. The Coronary Drug Project: Findings leading to discontinuation of the $2.5 \mathrm{mg} /$ day estrogen group. JAMA 1973; 226: 652-7.

${ }^{7}$ Henriksson P, Edhag O. Orchidectomy versus oestrogen for prostatic cancer: cardiovascular effects. $\mathrm{Br}$ Med $J$ 1986; 293: 413-5.

${ }^{8}$ Bailar JC. When research results are in conflict. $N$ Engl $J$ Med 1985; 313: 1080-1

${ }^{9}$ Stampfer MJ, Willett WC, Colditz GA, Rosner B, Speizer FE, Hennekens CH. A prospective study of postmenopausal estrogen therapy and coronary heart disease. N Engl J Med 1985; 313: 1044-9.

10 Wilson PWF, Garrison RJ, Castelli WP. Postmenopausal estrogen use, cigarette smoking and cardiovascular morbidity in women over 50: The Framingham Study. $N$ Engl J Med 1985; 313: 1038-43.

11 Meade TW, Greenberg G, Thompson SG. Progestogens and cardiovascular reactions associated with oral contraceptives and a comparison of the safety of 50 - and 30- $\mu$ g oestrogen preparations. $\mathrm{Br}$ Med $J$ 1980; 280: $1157-61$.

12 Kay CR. The happiness pill? J R Coll Gen Pract 1980; 30: 8-19.

${ }^{13}$ Vessey MP, Bungay GT. Benefits and risks of hormone therapy in the menopause. In: Smith A, ed. Recent advances in community medicine, No. 2. Edinburgh: Churchill Livingstone, 77-94.
${ }^{14}$ Medical Research Council's Working Party on mild to moderate hypertension. Randomized controlled trial of treatment of mild hypertension: design and pilot trial. $\mathrm{Br}$ Med J 1977; i: 1437-40.

15 World Health Organization Regional Office for Europe. Myocardial infarction community registers (Public Health in Europe No. 5). Copenhagen: WHO, 1976.

${ }^{16}$ Aho H, Harmsen P, Hatano S, Marquardsen J, Smirnov VE, Strasser T. Cerebrovascular disease in the community; results of a WHO collaborative study. Bull WHO 1980; 58: 113-30.

${ }^{17}$ Breslow NE, Day NE. Statistical methods in cancer research, vol 1: The analysis of case-control studies. Lyon: International Agency for Research on Cancer, 1980.

18 Greenberg G, Thompson SG, Meade TW. Relation between cigarette smoking and use of hormonal replacement therapy for menopausal symptoms. $J$ Epidemiol Community Health 1987; 41: 26-9.

${ }^{19}$ Stampfer MJ, Willett WC, Colditz GA, Rosner B, Speizer FE, Hennekens $\mathrm{CH}$. Postmenopausal estrogen use and heart disease (correspondence). $N$ Engl J Med 1986; 315: 135-6.

${ }^{20}$ Bush TL, Barrett-Connor E, Cowan LD, et al. Cardiovascular mortality and estrogen use: results from the Lipid Research Clinics Program Follow-up Study. Circulation 1987; 75: 1102-9.

${ }^{21}$ Bush TL, Barrett-Connor E. Noncontraceptive estrogen use and cardiovascular disease. Epidemiol Rev 1985; 7: 80-104.

22 Jick H, Dinan B, Rothman KJ. Noncontraceptive estrogens and nonfatal myocardial infarction. $J A M A$ 1978; 239: 1407-8.

23 Jick H, Dinan B, Herman R, Rothman KJ. Myocardial infarction and other vascular diseases in young women: role of estrogens and other factors. JAMA 1978; 240: 2548-52.

${ }^{24}$ Eaker ED, Castelli WP. Differential risk for coronary heart disease among women in the Framingham Study. In: Proceedings of the Workshop on Coronary Heart Disease in Women, Bethesda, Md., January 26-28th, 1986. 